\title{
DETERMINACION DE ELECTROLITOS EN LOS TRASTORNOS NUTRITIVOS AGUDOS
}

\author{
Por los Dres, OSCAR CORREA B. y ANDRES DEL VALLE \\ Hospital Luis Calvo Mackenna. Catedra de Pediatria del Prof, Anibal Ariztía.
}

Hecho de sobra conocido es la alta frecuencia y gravedad de los trastornos nutritivos agudos, dispepsia y toxicosis, en nuestro medio. Numerosos trabajos, presentados y discutidos tanto en el país como extranjero $0^{2}: 4$, testimonian la marcada y constante preocupación despertada por ellos.

A nadie escapa, desde los trabajos fun-

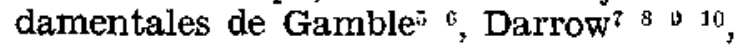
Hartmann ${ }^{11}$, Butler ${ }^{12}$, Fanconi ${ }^{13}{ }^{14}$ y otros, la importancia del estuđio de las alteraciones electrolíticas en los trastornos nutritivos agudos. Su conocimiento se hace cada día más indispensable para la correcta institución del tratamiento, constituído, en esencia, por el uso de diversas soluciones.

\section{Material y métodos.}

Nuestro estudio se realizó en los meses de Verano y Otoño de 1951 y en él incluímos lactantes aquejados de dispepsia o toxicosis de los Servicios de Lactantes y Recepción del Hóspital Luis Calvo Mackenna. Se reunieron en total 25 casos, que se agruparon en dos series, una compuesta de 16 (grupo A), en la que la terapéutica hidrosalina fué dirigida de acuerdo con las determinaciones de laboratorio y otra, de 9 (grupo B), cuyos componentes recibieron tratamiento sin conocer el médico las cifras de electrolitos. Salvo esto, los recursos terapéuticos para ambos grupos, incluído en ello el uso de antibióticos, fueron idénticos. Los exámenes comprendieron đeterminación đe sodio, potasio, cloro globular y plasmático, reserva alcalina y proteínas totales. Es de interés hacer presente que nuestro material sólo pudo ser constituído por lactantes en quienes fué posible cumplir satisfactoriamente varias condiciones de trabajo, entre ellas, extracción de sangre suficiente, no aireación de ésta, realización inmediata del examen, etc., todo lo cual es bien conocido para quienes tienen práctica en esta clase de estudios.

Salvo contados casos, en todos la sangre se obturo de la yugular interna. Se empleó aguja de $5 \mathrm{~cm}$ de largo y $1 \mathrm{~mm}$ de calibre y jeringa B-D, enfriada y seca, a fin de evitar hemolisis. Para las determinaciones de sodio y potasio, en que necesitábamos $7 \mathrm{~cm}^{3}$ de sangre, recibiamos ésta, de acuerdo con las recomendaciones de Corona" tubo de centrifuga, forma en que también procedíamos para reserva alcalina y cloremia, después de colocar algunos cristales de oxalato de $\mathrm{K}$ en el fondo del tubo. En este último caso depositábamos, mediante un tubo delgado, $6 \mathrm{~cm}^{3}$ de sangre debajo de 1 a $2 \mathrm{~cm}^{8}$ de vaselina líquida, obturábamos luego el extremo del tubo y agitábamos a fin de disolver el oxalato, precauciones que se hacen necesarias para evitar la aireación de la muestra. El sodio se determinó por el método basado en la precipitación del anión por el piroantimoniato de sodio, que es determinado por yodometría. Para el potasio se siguió la técnica de Kramer y para el cloro globular y plasmático y reserva alcalina ios conocidos métodos de Charpentier y Volhard y 
Van Slyke, respectivamente. La determinación de las proteínas totales del suero se hizo mediante el refractómetro de inmersión de Pulfrich.

\section{Plan terapéutico.}

Incluifo un caso en la experiencia se obtenía muestra de sangre y determinaba inmediatamente la reserva alcalina, lo, que permitia decidir sobre uso y dosis de la solución de Hartman.

Tratamiento de la acidosis: Frente a un valor bajo de reserva alcalina se usaba solución de Hartmann en partes iguales con suero glucosado al $10 \%$. En la mayoría de los niños empleamos la vía endovenosa y sólo en algunos de ellos, con buena tolerancia gástrica, la vía oral. Con el fin de calcular el volumen necesario de solución, seguimos el plan expuesto por Hand y Leininge ${ }^{16}$. El déficit de $\mathrm{CO}_{2}$ por litro es dado por la cifra de milequivalentes, la que resulta de la división del volumen de $\mathrm{CO}_{2} / \%$ por el factor 2.2. Si normalmente hay $27 \mathrm{mEq}$. y se han encontrado 43.3 vol. de $\mathrm{CO}_{2} \%$, o sea, $19 \mathrm{mEq}$., como sucede, por ejemplo, en el caso 1 , el déficit será de 8 mEq. (27-19). Ahora, si suponemos que la cantidad total de líquidos del organismo alcanza al $70 \%$ del peso corporal y el peso del lactante citado es de $5 \mathrm{~kg}$, habrá, en consecuencia, 3.5 litros de líquido total, de donde, por consiguiente, el déficit de $\mathrm{CO}_{\text {a }}$ será de 28 mEq. $(8 \times 3.5)$.

La composición de la solución alcalinizante de Hartmann se presenta en la Tabla 1. Un litro de esta solución proporciona $166.6 \mathrm{mEq}$. de álcalis, o sea, 6 cc contienen $1 \mathrm{mEq}$, de lo que se desprende que para restituir el déficit de $28 \mathrm{mEq}$. debemos suministrar $168 \mathrm{cc}$ de ella $(28 \times 6)$. Butler y Talbot estiman que puede llegar a cometerse un error de hasta el $50 \%$ en la apreciación de

Tabla 1

Contenido electrolitico de las soluciones empleadas. g por mil.

\begin{tabular}{|c|c|c|c|c|c|}
\hline Ringer: & $\mathbf{N a}$ & $\mathrm{Ca}$ & $\mathbf{K}$ & $\mathrm{Cl}$ & $\infty 2$ \\
\hline $\begin{array}{l}\mathrm{Na} \mathrm{Cl}-8.5 \mathrm{~g} \mathrm{o} / 00 \\
\mathrm{~K} \mathrm{Cl}-0.3 \mathrm{~g} \mathrm{o} / 00 \\
\mathrm{CaCl}-020 / 00 \mathrm{~g}\end{array}$ & $\stackrel{3.34}{-}$ & $\overline{-} \overline{0.072}$ & $\begin{array}{l}-\overline{157} \\
-\end{array}$ & $\begin{array}{l}5.16 \\
0.143 \\
0.128\end{array}$ & - \\
\hline SUMA & 3.34 & 0.072 & 0.157 & 5.432 & - \\
\hline $\begin{array}{l}\text { ce de la sol. que contíenen } 1 \mathrm{mEq} \text {. } \\
\text { mEq. por litro de solusión }\end{array}$ & $\begin{array}{c}7 \\
142.8\end{array}$ & $\begin{array}{r}277.7 \\
3.6\end{array}$ & $\begin{array}{r}250 \\
4\end{array}$ & $\begin{array}{r}6.5 \\
153.8\end{array}$ & - \\
\hline \multicolumn{6}{|l|}{ Darrow: } \\
\hline $\begin{array}{l}\mathrm{Na} \mathrm{HOO}-4.46 \mathrm{~g} \mathrm{O} / 00 \\
\mathrm{~K} \mathrm{Cl}-2.65 \mathrm{~g} \mathrm{o} / 00 \\
\mathrm{Na} \mathrm{Cl}-4.04 \mathrm{~g} \mathrm{o} / 00\end{array}$ & $\overline{1.22}$ & $\stackrel{-}{-}$ & $\overline{1.37}$ & $\begin{array}{l}-\overline{1.24} \\
2.46\end{array}$ & $\frac{2.45}{-}$ \\
\hline SUMA & 2.80 & - & 1.97 & 3.7 & $\begin{array}{r}2.45 \\
\ldots-\end{array}$ \\
\hline $\begin{array}{l}\text { cc de la sol, que contienen } 1 \mathrm{mEq} \text {. } \\
\mathrm{mEq} \text {. por litro de soluckón }\end{array}$ & $122^{8.2}$ & - & $\begin{array}{l}28.2 \\
35\end{array}$ & 105 & $\begin{array}{l}17.9 \\
55.9\end{array}$ \\
\hline Hartmann: & & & & $\cdot$ & $\begin{array}{l}\text { Ac, láctlco } \\
\text { (CO2) }\end{array}$ \\
\hline $\begin{array}{l}\text { Acido láctico } 15 \mathrm{~g} \text { o/00 } \\
\mathrm{Na} \text { OH }-2 \mathrm{~g} 0 / 00\end{array}$ & $\overline{1.15}$ & - & - & - & ${ }^{15}$ \\
\hline SƯMA & 1.15 & - & - & - & 15 \\
\hline $\begin{array}{l}\text { ce de la sol. que contienen } 1 \mathrm{mEa} \\
\mathrm{mEg} \text {, por litro de soluclon }\end{array}$ & $\begin{array}{l}50 \\
20\end{array}$ & - & - & - & $\begin{array}{c}6 \\
168.6\end{array}$ \\
\hline
\end{tabular}


los liquidos totales del organismo, por lo que recomiendan reemplazar sólo ia mitad del déficit. En los primeros casos que integran el estudio, nosotros seguimos esta conducta, pero, posteriormente, al comprobar que con tal proceder los valores de reserva alcalina quedaban lejos de la normalidad, decidimos restituir el déficit total, lo que se hizo sin que llegáramos a observar alcalosis.

Reemplazo de sodio y cloro: El déficit de sodio se determinó según el mismo procedimiento antes señalado, con la salvedad, se comprende, de hacerse el cálculo para un volumen líquido de sólo el $20 \%$ del peso del cuerpo, ya que este electrolito se encuentra casi exclusivamente en el líquido extracelular. Como solución de restitución usamos la de Ringer, 7 cc de la cual contienen $1 \mathrm{mEq}$. de $\mathrm{Na}$. En la determinación de la cantidad de $\mathrm{Na}$ por reemplazar se consideró también el $\mathrm{Na}$ incluído en la solución de Hartmann. El $\mathrm{Cl}$ se administró en general en exceso, ya que su déficit es proporcionalmente menor que el de $\mathrm{Na}$ y la solución de Ringer, empleada para corregir la deficiencia de éste, contiene cantidades cercanas de $\mathrm{Na}$ y Cl. Las soluciones de Ringer y Hartmann se usaron mezcladas en partes iguales con la de suero glucosado al $10 \%$, por via endovenosa.

Reemplazo de potasio: Este catión se administró en pequeña cantidad con la solución de Ringer y la solución de Darrow, que lo contiene en alta concentración, se reservó sólo para los casos con acentuado déficit de este electrolito, eventualidad en que se usó de acuerdo con la pauta aconsejada por Govan y Darrow ${ }^{17}$, que contempla la administración de 80 a 150 cc de solución por $\mathrm{kg}$ de peso el primer día y siempre que haya sido tratado el shock $y$ la función renal restablecida por el suministro de soluciones salinas y glucosadas. Los días siguientes pueden darse de 20 a 25 cc por $\mathrm{kg}$ de peso al día si las deposiciones acuosas se mantienen. A diferencia de los autores mencionados, que recomiendan administrar dicha solución por via hipodérmica, nosotros la indicamos por boca por el temor de observar intoxicaciones que por esta va nunca se han tenido $\mathrm{y}$, por el contrario, sí, y graves, por ia anterior.

Suministro de agua: A este respecto Cohen y Dubow ${ }^{\text {s }}$ aconsejan dar el primer día un volumen suficiente de agua para salvar el déficit de ella (calculado por la diferencia de peso), suplir los requerimientos de mantenimiento (100 a $150 \mathrm{cc}$ por $\mathrm{kg}$ de peso) y compensar las pérdioas anormales. En la mayoría de ios casos no nos fué posible determinar el volumen. de agua perdida por desconocer el peso anterior al trastorno nutritivo y en este punto sólo nos guiamos por el grado de deshidratación $\mathrm{y}$ el carácter y frecuencia de las deposiciones. En la mayor parte de los lactantes suministramos 200 a $300 \mathrm{cc}$ de agua por $\mathrm{kg}$ de peso el primer día, ya en soluciones como las antes mencionadas 0 en mezclas hidratantes dadas por vía oral, como se explicará a continuación.

Realimentación: Después de un período de dieta hídrica variable de 12 a 24 horas, lo que dependía de la gravedad del caso, se iniciaba la realimentación, que se ceñía a la pauta general en uso en el hospital y que contempla el empleo de una mezcla en partes iguales de suero de Babeurre (Eledón) y agua de arroz adicionada de $6 \%$ de maltosa-dextrina. Esta mezcla se da el primer dia a razón de $50 \mathrm{cc}$ por $\mathrm{kg}$ de peso corporal y posteriormente se aumenta en igual proporción o poco menos, según la respuesta del enfermo, hasta alcanzar al tercero o cuarto día a completar por si sola la ración líquida del día. Cuando así no ocurre esto se hace con una mezcla en partes iguales de suero Ringer y agua de canela con $6 \%$ de maltosa-dextrina, que es, por lo demás, la que también se usa durante el periodo de dieta hídrica exclusiva, en este caso sin agregado de maltosa-dextrina. A continuación se inicia el reemplazo de la mezcla hidratante por el alimento curativo, Babeurre o leche albuminosa con $5 \%$ de hidratos de carbono o leche humana, de los cuales se da $50 \mathrm{cc}$ por $\mathrm{kg}$ de peso el primer día y se aumenta después pro- 
gresivamente en igual forma que para la mezcla hidratante. Posteriormente, el niño es llevado poco a poco, en el plazo de una a dos semanas, al régimen normal para su edad.

Incluído un lactante en la experiencia y hechas las extracciones de sangre necesarias para las determinaciones antes mencionadas, se procedía, de acuerdo con los resultados arrojados por éstas, a indicar el tipo de solución a emplear así como el volumen de ésta. Después, al tercer día, se repetía la determinación de los electrolitos con el fin de mantener la orientación en el plan terapéutico de restitución. Durante todo este tiempo y a partir de la inclusion del paciente en el estudio, se llevaba un control cuidadoso diario del estado sensorial de éste (lucidez, obnubilación y apatía, inconsciencia), carácter de las respiraciones (acidótica, Cheyne-Stokes, etc.) y grado de deshidratación, ello juzgado por turgor y elasticidad de ia piel, estado de las mucosas, depresión de la fontanela, curva de peso, etc. Obtenida la recuperación clínica del paciente se practicaba un control final de electrolitos, que en algunos, por encontrarse cifras anormales, se repetía una o dos veces más.

\section{Resultados y comentario.}

No obstante el interés que hubiera podido revestir la presentación detallada del material, en obsequio a la brevedad reuniremos en tablas los principales datos del estudio. Más adelante, como ejemplo de la metódica seguida, expondremos separadamente algunas observaciones. La Tabla 2 se refiere al grupo de lactantes en que la terapéutica de restitución hidrosalina fué dirigida de acuerdo con los resultados del laboratorio y la Tabla 3 a la de aquellos que recibieron tratamiento sin darse a conocer al personal tratante los informes de éste.

Si analizamos las determinaciones de $\mathrm{Na}$ encontraremos que de los 23 casos en que este examen se hizo en 19 se comprobó un valor bajo; en 3, elevado y en 2, normal. Las cifras extremas fluctuaron entre 80 y $152 \mathrm{mEq}$. Estos resultados se asemejan a los obtenidos por del Carril y Larguía ${ }^{10}$, autores que encontraron en 36 casos 21 con cifras bajo $140 \mathrm{mEq}$., 6 sobre $160 \mathrm{mEq}$. y 9 entre 140 y $160 \mathrm{mEq}$. El alto porcentaje de natremias bajas en nuestro material concuerda con el conocimiento ya clásico de la pérdida de este anión en los trastornos nutritivos agudos. En nuestro estudio no comprobamos paralelismo entre el grado de deshidratación $y$ la intensidad del déficit de $\mathrm{Na}$, como se evidencia muy bien en los casos 10 y 21 , en los que cifras decididamente bajas, alrededor de $90 \mathrm{mEq}$., coinciden en el primero con deshidratación ligera y en el segundo con deshidratación extrema.

En cuanto a la reserva alcalina, de 25 casos en que se determinó, en 22 resultó inferior a $23 \mathrm{mEq}$. (50 vol. de CO. $\%$ ) y en 3 , normal, siendo el valor más bajo encontrado $5 \mathrm{mEq}$. (11 vol. de $\mathrm{CO}_{2} \%$ ) y el más alto, $26 \mathrm{mEq}$. (57.2 vol. de $\mathrm{CO}_{2} \%$ ). De los 15 lactantes con toxicosis estudiados en todos encontramos disminución acentuada del bicarbonato. Si relacionamos las manifestaciones cilnicas con los valores de reserva alcalina cabe suponer en 21 del total de 25 casos la existencia. de un estado de acídosis no gaseosa (confirmada en 13 por aumento del índice clorémico), en 2, normalidad del equilibrio ácido-básico $\mathrm{y}$ en 1 (caso 14), alcalosis gaseosa. Del Carril y Larguía dan cuenta de resultados parecidos al expresar que en el $90 \%$ de sus pacientes encontraron acidosis; en $8 \%$, reserva alcalina normal $\mathrm{y}$ en el $2 \%$, alcalosis gaseosa. Un punto interesante que cabe recalcar a propósito de este examen es que de los 21 lactantes en que el laboratorio señaló la existencía de acidosis sólo en 9 de ellos, con cifras de bicarbonato entre 8 y $20 \mathrm{mEq}$., el clínico pudo sospechar dicho estado por el carácter de las respiraciones, no siendo evidente en los restantes este sig. no ni aun con cifras de aquél extremadamente bajas. 
Tabla 2 - Grupo A.

\begin{tabular}{|c|c|c|c|c|c|c|c|c|c|c|c|c|c|c|c|}
\hline $\mathbf{N}$ & $\begin{array}{l}\text { Nombre } \\
\text { Edad } \\
\text { Obs. }\end{array}$ & Diagnóstico & Día & $\begin{array}{c}\text { Peso } \\
\text { g }\end{array}$ & $\begin{array}{l}\text { Con- } \\
\text { cienc. }\end{array}$ & $\begin{array}{l}\text { Des- } \\
\text { hidr. }\end{array}$ & $\begin{array}{c}\mathrm{Na} \\
\mathrm{mEq}\end{array}$ & $\underset{\mathrm{mE}}{\mathrm{K}}$ & $\begin{array}{c}\mathrm{H} \mathrm{COS} \\
\text { mEq. }\end{array}$ & $\begin{array}{l}\mathrm{ClG} \\
\text { miEq. }\end{array}$ & $\begin{array}{l}\text { ClP } \\
\text { mEq. }\end{array}$ & $\begin{array}{l}\text { Indice } \\
\text { clorémico }\end{array}$ & $\begin{array}{c}\text { Protein. } \\
\mathrm{g} \mathrm{O} / 00\end{array}$ & $\begin{array}{l}\text { Hemato- } \\
\text { crito } \%\end{array}$ & $\begin{array}{l}\mathbf{H b} . \\
\mathbf{g} \% \overline{i /}\end{array}$ \\
\hline 1. & $\begin{array}{l}\text { P. B. } \\
\text { 5 meses } \\
51-2000\end{array}$ & $\begin{array}{l}\mathrm{Dt} \\
\mathrm{Dp}\end{array}$ & $\begin{array}{l}1 \\
3 \\
7\end{array}$ & $\begin{array}{l}4960 \\
5280 \\
5320\end{array}$ & $\begin{array}{l}\mathrm{N} \\
\mathrm{N} \\
\mathrm{N}\end{array}$ & $\begin{array}{l}2 \\
\mathrm{H} \\
\mathrm{H}\end{array}$ & $\begin{array}{l}113 \\
121 \\
126\end{array}$ & $\begin{array}{r}4.6 \\
7.6 \\
10.0\end{array}$ & $\begin{array}{l}19 \\
25 \\
21\end{array}$ & $\begin{array}{l}54 \\
51 \\
51\end{array}$ & $\begin{array}{r}107 \\
90 \\
89\end{array}$ & $\begin{array}{l}0.50 \\
0.56 \\
0.51\end{array}$ & $\begin{array}{l}54.5 \\
48.1 \\
61.2\end{array}$ & $\frac{-}{39.5}$ & $\overline{12.0}$ \\
\hline 2. & $\begin{array}{l}\text { D. C. } \\
9 \text { meses } \\
51-2288\end{array}$ & $\begin{array}{l}\mathbf{D t} \\
\mathbf{T} \\
\mathbf{D t}\end{array}$ & $\begin{array}{r}1 \\
3 \\
6 \\
15\end{array}$ & $\begin{array}{l}4560 \\
5100 \\
5200 \\
5720\end{array}$ & $\begin{array}{c}\mathbf{S}-\mathbf{I} \\
\mathbf{N} \\
\mathbf{N} \\
\mathbf{N}\end{array}$ & $\begin{array}{l}3 \\
1 \\
\mathrm{H} \\
\mathrm{H}\end{array}$ & $\begin{array}{l}104 \\
130 \\
140 \\
130\end{array}$ & $\begin{array}{l}6.6 \\
9.0 \\
6.1 \\
5.0\end{array}$ & $\begin{array}{r}8 \\
13 \\
19 \\
16\end{array}$ & $\begin{array}{l}54 \\
51 \\
54 \\
56\end{array}$ & $\begin{array}{r}102 \\
102 \\
99 \\
98\end{array}$ & $\begin{array}{l}0.52 \\
0.52 \\
0.54 \\
0.57\end{array}$ & $\begin{array}{l}80.6 \\
59.0 \\
46.0 \\
67.7\end{array}$ & $\begin{array}{c}41.5 \\
-- \\
- \\
-\end{array}$ & $\begin{array}{r}13.3 \\
- \\
-\end{array}$ \\
\hline 3. & $\begin{array}{l}\text { M. G. } \\
\mathbf{3 , 5} \text { meses } \\
51-819\end{array}$ & $\mathrm{~T}$ & $\begin{array}{l}1 \\
3 \\
7\end{array}$ & $\begin{array}{l}4620 \\
4800 \\
4800\end{array}$ & $\begin{array}{c}\mathrm{Ob} . \\
\mathrm{N} \\
\mathbf{N}\end{array}$ & $\begin{array}{l}3 \\
\mathbf{H} \\
\mathbf{H}\end{array}$ & $\begin{array}{l}130 \\
113 \\
125\end{array}$ & $\begin{array}{l}5.8 \\
7.6 \\
7.6\end{array}$ & $\begin{array}{r}8 \\
13 \\
18\end{array}$ & $\begin{array}{l}73 \\
70 \\
54\end{array}$ & $\begin{array}{r}110 \\
115 \\
99\end{array}$ & $\begin{array}{l}0.66 \\
0.60 \\
0.54\end{array}$ & $\begin{array}{l}88.2 \\
61.2 \\
61.2\end{array}$ & $\begin{array}{c}36.0 \\
-\end{array}$ & $\begin{array}{l}11.1 \\
-\end{array}$ \\
\hline 4. & $\begin{array}{l}\text { M. V. } \\
3,5 \text { meses } \\
51-202\end{array}$ & $\stackrel{A}{\mathrm{D} p}$ & $\begin{array}{l}1 \\
3 \\
7\end{array}$ & $\begin{array}{c}3800 \\
4250 \\
4530\end{array}$ & $\begin{array}{l}\mathbf{A} \\
\mathbf{N} \\
\mathrm{N}\end{array}$ & $\begin{array}{c}3-3 \\
2 \\
H\end{array}$ & $\begin{array}{l}120 \\
108 \\
120\end{array}$ & $\frac{2.8}{5.0}$ & $\begin{array}{l}16 \\
26 \\
23\end{array}$ & $\begin{array}{l}- \\
-\end{array}$ & $\begin{array}{r}104 \\
101 \\
93\end{array}$ & $\overline{-}$ & $\begin{array}{l}- \\
-\end{array}$ & $\begin{array}{c}32.0 \\
- \\
-\end{array}$ & $\begin{array}{c}11.9 \\
- \\
-\end{array}$ \\
\hline 5. & $\begin{array}{l}\text { M. M. } \\
7 \text { meses } \\
50-5147\end{array}$ & $\begin{array}{l}\mathrm{Dt} \\
\mathrm{Dp}\end{array}$ & $\begin{array}{r}1 \\
3 \\
7 \\
17\end{array}$ & $\begin{array}{l}4240 \\
4300 \\
4650 \\
4700\end{array}$ & $\begin{array}{l}\mathrm{A} \\
\mathrm{N} \\
\mathrm{N} \\
\mathrm{N}\end{array}$ & $\begin{array}{l}1 \\
\mathbf{H} \\
\mathbf{H} \\
\mathbf{H}\end{array}$ & $\begin{array}{l}120 \\
130 \\
126 \\
123\end{array}$ & $\begin{array}{r}6.0 \\
10.0 \\
12.0 \\
9.0\end{array}$ & $\begin{array}{l}25 \\
25 \\
20 \\
11\end{array}$ & $\begin{array}{l}48 \\
33 \\
54 \\
54\end{array}$ & $\begin{array}{r}93 \\
93 \\
102 \\
104\end{array}$ & $\begin{array}{l}0.53 \\
0.40 \\
0.52 \\
0.51\end{array}$ & $\begin{array}{l}59.0 \\
54.7 \\
48.1 \\
59.0\end{array}$ & $\begin{array}{c}38.0 \\
- \\
-\end{array}$ & $\begin{array}{r}11.9 \\
- \\
-\end{array}$ \\
\hline 6. & $\begin{array}{l}\text { H. G. } \\
3 \text { años } \\
49-3968\end{array}$ & Dt & $\begin{array}{l}1 \\
3 \\
4\end{array}$ & $\begin{array}{c}9800 \\
10120 \\
\text { Fallecío }\end{array}$ & $\stackrel{\mathrm{Ob} .}{\mathrm{N}}$ & $\begin{array}{l}2 \\
1\end{array}$ & $\begin{array}{r}80 \\
104\end{array}$ & $\begin{array}{l}2.0 \\
2.0\end{array}$ & $\begin{array}{l}25 \\
25\end{array}$ & - & $\begin{array}{l}76 \\
89\end{array}$ & - & $\overline{46.0}$ & - & $\overline{-}$ \\
\hline 7. & $\begin{array}{l}F . V . \\
6 \text { meses } \\
50-3552\end{array}$ & $\begin{array}{l}\mathbf{D} t \\
\mathbf{T}\end{array}$ & $\begin{array}{l}1 \\
3 \\
7\end{array}$ & $\begin{array}{l}\mathbf{5 5 2 0} \\
\mathbf{5 5 2 0} \\
\mathbf{6 2 0 0}\end{array}$ & $\begin{array}{c}\text { S-I } \\
\mathbf{N} \\
\mathbf{N}\end{array}$ & $\begin{array}{l}2 \\
\mathrm{H} \\
\mathrm{H}\end{array}$ & $\begin{array}{l}108 \\
126 \\
173\end{array}$ & $\begin{array}{l}\overline{4.6} \\
6.6\end{array}$ & $\begin{array}{l}14.5 \\
21.0 \\
26.0\end{array}$ & - & $\begin{array}{r}99 \\
101 \\
102\end{array}$ & $\overline{-}$ & $\begin{array}{c}73.1 \\
- \\
-\infty\end{array}$ & $\begin{array}{c}38.0 \\
- \\
-\end{array}$ & $\begin{array}{c}12.8 \\
-\end{array}$ \\
\hline 8. & $\begin{array}{l}\text { A. B. } \\
2 \text { meses } \\
51-1243\end{array}$ & $\begin{array}{l}\mathrm{A} \\
\mathrm{Dp}\end{array}$ & $\begin{array}{l}1 \\
3 \\
8\end{array}$ & $\begin{array}{l}3140 \\
3490 \\
3560\end{array}$ & $\begin{array}{l}\mathbf{A} \\
\mathbf{N} \\
\mathbf{N}\end{array}$ & $\begin{array}{c}2-3 \\
2 \\
\mathrm{H}\end{array}$ & $\begin{array}{l}152 \\
140 \\
130\end{array}$ & $\begin{array}{l}5.3 \\
5.3 \\
8.9\end{array}$ & $\begin{array}{l}15 \\
17.5 \\
26\end{array}$ & $\begin{array}{l}79 \\
59 \\
54\end{array}$ & $\begin{array}{r}121 \\
104 \\
98\end{array}$ & $\begin{array}{l}0.65 \\
0.56 \\
0.54\end{array}$ & $\begin{array}{l}76.3 \\
52.5 \\
5.8 .0\end{array}$ & $\frac{39}{-}$ & $\begin{array}{c}12.8 \\
- \\
-\end{array}$ \\
\hline 9. & $\begin{array}{l}\text { P. A. } \\
4 \text { meses } \\
51-1893\end{array}$ & $\begin{array}{l}\mathrm{Dt} \\
\mathrm{Dp}\end{array}$ & $\begin{array}{l}1 \\
3 \\
7\end{array}$ & $\begin{array}{l}4400 \\
4500 \\
4540\end{array}$ & $\begin{array}{c}\text { Ob. } \\
\mathbf{N} \\
\mathbf{N}\end{array}$ & $\begin{array}{c}2-3 \\
2 \\
\mathbf{H}\end{array}$ & $\begin{array}{l}108 \\
134 \\
143\end{array}$ & $\begin{array}{l}7 \\
6.4 \\
9\end{array}$ & $\begin{array}{l}21 \\
19 \\
24\end{array}$ & $\begin{array}{l}51 \\
45 \\
51\end{array}$ & $\begin{array}{l}84 \\
84 \\
98\end{array}$ & $\begin{array}{l}0.50 \\
0.53 \\
0.57\end{array}$ & $\frac{82.8}{68.7}$ & $\begin{array}{l}- \\
-\end{array}$ & - \\
\hline 10. & $\begin{array}{l}\text { M. L. } \\
6 \text { meses } \\
51-79\end{array}$ & $\begin{array}{l}\mathrm{Dt} \\
\mathrm{T}\end{array}$ & $\begin{array}{l}1 \\
\mathbf{5} \\
6 \\
9\end{array}$ & $\begin{array}{l}4650 \\
4690 \\
4630 \\
4722\end{array}$ & $\begin{array}{c}\text { Ob. } \\
\text { N } \\
\mathbf{N} \\
\mathbf{N}\end{array}$ & $\begin{array}{l}1 \\
1 \\
1 \\
H\end{array}$ & $\begin{array}{r}91 \\
95 \\
\frac{1}{126}\end{array}$ & $\begin{array}{c}0.8 \\
2.5 \\
- \\
-\end{array}$ & $\begin{array}{l}11 \\
26.6 \\
24\end{array}$ & $\begin{array}{l}54 \\
- \\
-\end{array}$ & $\begin{array}{l}95 \\
87 \\
-91\end{array}$ & $\begin{array}{c}0.55 \\
- \\
-- \\
--\end{array}$ & $\begin{array}{l}- \\
- \\
-\end{array}$ & $\frac{-}{33}$ & $\overline{11.9}$ \\
\hline
\end{tabular}




\begin{tabular}{|c|c|c|c|c|c|c|c|c|c|c|c|c|c|c|c|}
\hline & & & & & & & & & & & & & & & \\
\hline $\mathbf{N}^{\prime \prime}$ & $\begin{array}{c}\text { Nomibre } \\
\text { Edad } \\
\text { Obs. }\end{array}$ & Diagnóstico & Dia & $\begin{array}{c}\text { Peso } \\
\text { g }\end{array}$ & $\begin{array}{l}\text { Con- } \\
\text { cienc. }\end{array}$ & $\begin{array}{l}\text { Des- } \\
\text { hidr. }\end{array}$ & $\begin{array}{c}\mathrm{Na} \\
\mathrm{mE} \mathrm{F}\end{array}$ & $\underset{\mathrm{mEq}}{\mathrm{K}}$ & $\begin{array}{c}\mathrm{H} \cos 3 \\
\mathrm{mEq}\end{array}$ & $\begin{array}{l}\mathrm{Cl} \mathrm{G} \\
\mathrm{mSq}\end{array}$ & $\begin{array}{l}\mathrm{Cl} \mathrm{P} \\
\mathrm{mEq}\end{array}$ & $\begin{array}{c}\text { Indice } \\
\text { clorémico }\end{array}$ & $\begin{array}{c}\text { Proteín. } \\
\text { g o/oo }\end{array}$ & $\begin{array}{l}\text { Hemato- } \\
\text { crito } \%\end{array}$ & $\begin{array}{l}\mathrm{Hb} \\
\mathrm{g} \cdot \mathrm{r}\end{array}$ \\
\hline 21. & $\begin{array}{l}\text { J. C. } \\
3 \text { meses } \\
50-6733\end{array}$ & $\begin{array}{l}\mathbf{D t} \\
\mathbf{T}\end{array}$ & $\begin{array}{l}1 \\
2 \\
3\end{array}$ & $\begin{array}{l}4400 \\
4730 \\
4400\end{array}$ & $\begin{array}{l}\text { I } \\
\text { I } \\
\text { I }\end{array}$ & $\begin{array}{c}2 \\
2-1 \\
2-1\end{array}$ & $\begin{array}{c}\overline{126} \\
\text { Falleció }\end{array}$ & 3 & $\begin{array}{l}12 \\
15 \\
-\end{array}$ & - & $\begin{array}{r}\overline{104} \\
-\end{array}$ & $\begin{array}{l}- \\
-- \\
-\end{array}$ & $\frac{-}{64.3}$ & $\overline{35}$ & $\overline{\overline{1}}$ \\
\hline $\begin{array}{l}12 . \\
*\end{array}$ & $\begin{array}{l}\text { C. S. } \\
7 \text { meses } \\
51-1750\end{array}$ & $\begin{array}{l}\mathrm{Dt} \\
\mathbf{T}\end{array}$ & $\begin{array}{r}1 \\
2 \\
3 \\
7 \\
12 \\
16\end{array}$ & $\begin{array}{l}61000 \\
6320 \\
6300 \\
6300 \\
6400 \\
6670\end{array}$ & $\begin{array}{l}\text { Ob. } \\
\mathbf{N} \\
\mathbf{N} \\
\mathbf{N} \\
\mathbf{N} \\
\mathbf{N}\end{array}$ & $\begin{array}{l}2 \\
1 \\
1 \\
1 \\
1 \\
\text { H }\end{array}$ & $\begin{array}{l}147 \\
\overrightarrow{113} \\
130 \\
180 \\
113\end{array}$ & $\begin{array}{l}3 \\
7.6 \\
5.6 \\
3 \\
6.6\end{array}$ & $\begin{array}{l}13 \\
19 \\
25 \\
24 \\
27 \\
23\end{array}$ & $\begin{array}{l}\frac{56}{59} \\
51 \\
\frac{51}{51}\end{array}$ & $\begin{array}{r}90 \\
-93 \\
102 \\
96 \\
87\end{array}$ & $\begin{array}{c}0.62 \\
-\overline{63} \\
0.63 \\
0.50 \\
\overline{0.54}\end{array}$ & $\begin{array}{c}80.6 \\
- \\
57.9 \\
52.5 \\
50.3 \\
52.5\end{array}$ & $\begin{array}{l}44 \\
= \\
- \\
-\end{array}$ & $\begin{array}{l}14.5 \\
- \\
- \\
-\end{array}$ \\
\hline $\begin{array}{l}13 . \\
*\end{array}$ & $\begin{array}{l}\text { S. R. } \\
3 \text { meses } \\
50-5191\end{array}$ & $\begin{array}{l}\mathbf{A} \\
\mathbf{T}\end{array}$ & $\begin{array}{l}1 \\
2 \\
3 \\
9\end{array}$ & $\begin{array}{l}2550 \\
2750 \\
2750 \\
2960\end{array}$ & $\begin{array}{c}\text { Ob. } \\
\mathbf{N} \\
\mathbf{N} \\
\mathbf{N}\end{array}$ & $\begin{array}{l}3 \\
2 \\
2 \\
\text { H }\end{array}$ & $\begin{array}{l}147 \\
126 \\
147\end{array}$ & $\begin{array}{l}- \\
\overline{3.5} \\
5.6\end{array}$ & $\begin{array}{l}15 \\
13 \\
14 \\
27\end{array}$ & $\begin{array}{l}- \\
- \\
-\end{array}$ & $\begin{array}{r}104 \\
110 \\
104\end{array}$ & $\begin{array}{l}- \\
\overline{-}\end{array}$ & $\begin{array}{c}5 \overline{-} \\
- \\
-\end{array}$ & $\begin{array}{l}27 \\
-\end{array}$ & $\begin{array}{l}\frac{-}{9.4} \\
\frac{-4}{9.4}\end{array}$ \\
\hline 14. & $\begin{array}{l}\text { J. B. } \\
4 \text { meses } \\
51-758\end{array}$ & $\underset{\mathrm{T}}{\mathrm{A}}$ & 1 & $\begin{array}{l}3280 \\
\text { Fallecic }\end{array}$ & $o^{I}$ & 3 & - & - & 5 & 67 & 135 & 0.48 & - & 27.6 & 11 \\
\hline 15. & $\begin{array}{l}\text { J. M. } \\
5 \text { meses } \\
51-55\end{array}$ & $\begin{array}{l}\mathbf{D t} \\
\mathbf{T}\end{array}$ & $\begin{array}{l}1 \\
\mathbf{3} \\
\mathbf{8}\end{array}$ & $\begin{array}{l}4400 \\
4500 \\
4670\end{array}$ & $\begin{array}{l}\mathrm{Ob} . \\
\mathbf{N} \\
\mathbf{N}\end{array}$ & $\begin{array}{l}2 \\
\mathrm{H} \\
\mathrm{H}\end{array}$ & $\begin{array}{r}107 \\
\cdot 113 \\
126\end{array}$ & $\begin{array}{l}2.3 \\
2.6 \\
3\end{array}$ & $\begin{array}{r}9 \\
24 \\
23\end{array}$ & $\frac{62}{-}$ & $\begin{array}{r}101 \\
101 \\
99\end{array}$ & $\frac{0.31}{-}$ & $\frac{-}{43.8}$ & 30 & $\overline{11.1}$ \\
\hline 16. & $\begin{array}{l}\text { M. M. } \\
\text { 4,5 meses } \\
51-25=8\end{array}$ & s $\quad \begin{array}{l}A \\
\mathrm{Dp}\end{array}$ & $\begin{array}{l}1 \\
\mathbf{3} \\
\mathbf{8}\end{array}$ & $\begin{array}{l}3340 \\
3420 \\
3580\end{array}$ & $\begin{array}{c}\mathrm{Ob} . \\
\mathrm{N} \\
\mathrm{N}\end{array}$ & $\begin{array}{c}2-3 \\
2 \\
1\end{array}$ & $\begin{array}{l}113 \\
126 \\
140\end{array}$ & $\begin{array}{l}3.5 \\
2.8 \\
5.3\end{array}$ & $\begin{array}{l}10.5 \\
17 \\
17\end{array}$ & $\begin{array}{l}54 \\
51 \\
54\end{array}$ & $\begin{array}{r}87 \\
96 \\
104\end{array}$ & $\begin{array}{l}0.61 \\
0.52 \\
0.51\end{array}$ & $\begin{array}{l}68.8 \\
63.4 \\
63.4\end{array}$ & $\begin{array}{l}46 \\
-\end{array}$ & $\begin{array}{c}13.3 \\
-\end{array}$ \\
\hline
\end{tabular}

Los casos señalados con $*$ tenían respiraciones profundas al ingreso.
Fil caso 6, una niñita de 3 años, se incluye por haber presentado un cuadro de dlarrea grave con intensa deshiơratación y embotamiento sensorial en todo semejante a la toxicosis del lactante.

Expliceción de atreviaturas:

Diagnóstico: Dt: distrofia, Dp: dispepsia, A: atrotia, $\mathbf{T}$ : toxicosis.

Consclencia: $\mathbf{N}$ : normal. A: apatia, Ob: obnubilaclón, S-I: seml-fnconssiencia, I: inconsciencla.

Deshidratación: Grado 1: mucosas húmedas y pérdida del turgor. Grado 2: mucosas secas y turgor muy disminuido. Grado 3 : deshidratación extrema con persistencia de pliegues y escleredema. H: hidratación normal. 
Tabla 3 - Grupo $\mathbf{B}$

\begin{tabular}{|c|c|c|c|c|c|c|c|c|c|c|c|c|c|c|c|}
\hline $\mathbf{N}^{\prime}$ & $\begin{array}{l}\text { Nombre } \\
\text { Edaut } \\
\text { Obs. }\end{array}$ & Diagnóstico & Dia & $\begin{array}{l}\text { Peso } \\
\text { g }\end{array}$ & $\begin{array}{l}\text { Con- } \\
\text { clenc. }\end{array}$ & $\begin{array}{l}\text { Des- } \\
\text { hidr. }\end{array}$ & $\begin{array}{l}\mathrm{Na} \\
\mathrm{mEq}\end{array}$ & $\underset{\mathrm{mEq}}{\mathbf{K}}$ & $\begin{array}{c}\text { H CiO3 } \\
\text { mEq. }\end{array}$ & $\begin{array}{l}\mathrm{ClC} \\
\mathrm{mEq}\end{array}$ & $\begin{array}{l}\mathrm{Cl} \mathbf{P} \\
\mathrm{mEq}\end{array}$ & $\begin{array}{l}\text { Indice } \\
\text { clarémico }\end{array}$ & $\begin{array}{c}\text { Protein. } \\
\mathrm{g} 0 / 00\end{array}$ & $\begin{array}{l}\text { Hemato- } \\
\text { crito } \%\end{array}$ & $\begin{array}{l}\text { Hi. } \\
\text { g \% }\end{array}$ \\
\hline 17. & $\begin{array}{l}\text { M. M. } \\
9 \text { meses } \\
51-503\end{array}$ & $\begin{array}{l}\mathrm{Dt} \\
\mathrm{Dp}\end{array}$ & $\begin{array}{l}1 \\
8 \\
3 \\
8\end{array}$ & $\begin{array}{c}5750 \\
5900 \\
- \\
-\end{array}$ & $\begin{array}{l}\mathrm{N} \\
\mathrm{N} \\
\mathrm{N} \\
\mathrm{N}\end{array}$ & $\begin{array}{l}2 \\
2 \\
1 \\
\mathbf{H}\end{array}$ & $\begin{array}{c}117 \\
173 \\
126\end{array}$ & $\frac{8.4}{3.2}$ & $\begin{array}{l}\frac{21}{24} \\
19.6\end{array}$ & $\begin{array}{l}- \\
- \\
-\end{array}$ & $\begin{array}{l}94 \\
\cdot- \\
97.5 \\
82\end{array}$ & $\begin{array}{l}- \\
-- \\
-\end{array}$ & $\begin{array}{r}52.5 \\
- \\
-\end{array}$ & $\begin{array}{l}-- \\
45 \\
-\end{array}$ & $\frac{-}{13.8}$ \\
\hline $\begin{array}{l}18 . \\
+\end{array}$ & $\begin{array}{l}\mathbf{E}, \mathbf{B} . \\
8 \text { meses } \\
51-647\end{array}$ & $\begin{array}{l}\mathrm{Dt} \\
\mathrm{T}\end{array}$ & $\begin{array}{l}1 \\
3 \\
8\end{array}$ & $\begin{array}{l}4750 \\
4700 \\
5200\end{array}$ & $\underset{\mathbf{S}-I}{\mathrm{I}}$ & $\begin{array}{c}3 \\
2-3 \\
2-1\end{array}$ & $\begin{array}{l}122 \\
114 \\
101\end{array}$ & $\begin{array}{l}8 \\
4 \\
5.6\end{array}$ & $\begin{array}{l}8 \\
16.6 \\
17\end{array}$ & $\begin{array}{l}51 \\
45 \\
42\end{array}$ & $\begin{array}{l}82 \\
91 \\
96\end{array}$ & $\begin{array}{l}0.64 \\
0.50 \\
0.42\end{array}$ & $\begin{array}{c}68.8 \\
56.8 \\
-\end{array}$ & $\begin{array}{l}40 \\
-- \\
--\end{array}$ & $\begin{array}{c}12.8 \\
\cdots \\
--\end{array}$ \\
\hline 19. & $\begin{array}{l}\text { A. S. } \\
\mathbf{2} \text { meses } \\
\mathbf{5 1 - 1 1 7}\end{array}$ & $\begin{array}{l}\mathbf{D t} \\
\mathbf{T}\end{array}$ & $\begin{array}{l}1 \\
3 \\
8\end{array}$ & $\begin{array}{l}3400 \\
3440 \\
3550\end{array}$ & $\begin{array}{l}\text { A } \\
\text { A } \\
\text { A }\end{array}$ & $\begin{array}{l}2 \\
2 \\
2\end{array}$ & $\begin{array}{l}140 \\
124 \\
134\end{array}$ & $\begin{array}{l}5.8 \\
2.8 \\
4\end{array}$ & $\begin{array}{c}8 \\
7 \\
11.4\end{array}$ & $\frac{59}{42}$ & $\begin{array}{r}102 \\
101 \\
96\end{array}$ & $\frac{0.58}{0.42}$ & $\begin{array}{c}74.2 \\
- \\
-\end{array}$ & $\frac{32}{-}$ & $\begin{array}{c}10.5 \\
-\end{array}$ \\
\hline 20. & $\begin{array}{l}\text { C. A. } \\
11 \text { meses } \\
50-3302\end{array}$ & $\begin{array}{l}\text { Dt } \\
\text { Dp }\end{array}$ & $\begin{array}{l}1 \\
3 \\
8\end{array}$ & $\begin{array}{l}5200 \\
5280 \\
5040\end{array}$ & $\begin{array}{l}\mathbf{N} \\
\mathbf{N} \\
\mathbf{N}\end{array}$ & $\begin{array}{l}2 \\
1 \\
1\end{array}$ & $\begin{array}{l}140 \\
120 \\
125\end{array}$ & $\begin{array}{l}1.4 \\
9 \\
4.6\end{array}$ & $\begin{array}{l}15.7 \\
16.3 \\
18\end{array}$ & $\frac{51}{33}$ & $\begin{array}{r}102 \\
100 \\
93\end{array}$ & $\frac{0.50}{0.39}$ & $\begin{array}{c}59.0 \\
- \\
-\end{array}$ & $\frac{43}{-}$ & $\begin{array}{c}13.3 \\
- \\
-\end{array}$ \\
\hline 21 & $\begin{array}{l}\text { A. C. } \\
10 \text { meses } \\
51-804\end{array}$ & $\begin{array}{l}\mathrm{Dt} \\
\mathrm{T}\end{array}$ & 1 & $\begin{array}{l}5210 \\
\text { Falleció }\end{array}$ & $\begin{array}{l}\mathbf{I} \\
6\end{array}$ & 3 & 92 & 2.8 & 5 & 62 & 118 & 0.52 & 80.6 & 59 & 17.6 \\
\hline 22. & $\begin{array}{l}\text { T. C. } \\
4 \text { meses } \\
51-565\end{array}$ & $\begin{array}{l}\mathrm{Dt} \\
\mathrm{T}\end{array}$ & $\begin{array}{l}1 \\
2 \\
4 \\
9\end{array}$ & $\begin{array}{l}4100 \\
4000 \\
4160 \\
4460\end{array}$ & $\begin{array}{l}\mathbf{N} \\
\mathbf{N} \\
\mathbf{N} \\
\mathbf{N}\end{array}$ & $\begin{array}{c}2 \\
2 \\
2-1 \\
1\end{array}$ & $\begin{array}{l}- \\
126 \\
130 \\
150\end{array}$ & $\begin{array}{l}- \\
6 \\
4.6 \\
6\end{array}$ & $\begin{array}{l}1.5 \\
18.3 \\
20 \\
20\end{array}$ & $\begin{array}{l}- \\
42 \\
48\end{array}$ & $\begin{array}{r}- \\
101 \\
94 \\
70\end{array}$ & $\begin{array}{l}- \\
\overline{1.45} \\
0.73\end{array}$ & $\begin{array}{l}- \\
- \\
-\end{array}$ & $\begin{array}{l}- \\
- \\
-\end{array}$ & $\begin{array}{l}- \\
- \\
-\end{array}$ \\
\hline 23. & $\begin{array}{l}\text { M. F. } \\
\text { 10 meses } \\
51-425\end{array}$ & $\begin{array}{l}\text { Dt } \\
\text { Dp }\end{array}$ & $\begin{array}{r}1 \\
2 \\
3 \\
6 \\
8 \\
15\end{array}$ & $\begin{array}{l}4600 \\
4600 \\
4600 \\
4700 \\
4700 \\
4800\end{array}$ & $\begin{array}{l}\mathrm{N} \\
\mathbf{N} \\
\mathbf{N} \\
\mathbf{N} \\
\mathbf{N} \\
\mathbf{N}\end{array}$ & $\begin{array}{l}1 \\
1 \\
1 \\
H \\
H \\
H\end{array}$ & $\begin{array}{r}104 \\
\overline{130} \\
135 \\
130 \\
170\end{array}$ & $\begin{array}{l}5.6 \\
\frac{4}{-} \\
8.5 \\
5\end{array}$ & $\begin{array}{l}24 \\
31 \\
39 \\
39 \\
17.4\end{array}$ & $\begin{array}{l}- \\
\frac{-}{51}\end{array}$ & $\begin{array}{c}87.5 \\
- \\
85 \\
85 \\
86 \\
97\end{array}$ & $\begin{array}{l}-- \\
-- \\
- \\
- \\
-52\end{array}$ & $\begin{array}{c}-\overline{56.8} \\
- \\
- \\
- \\
-\end{array}$ & $\begin{array}{l}27 \\
- \\
- \\
- \\
--\end{array}$ & $\begin{array}{l}10 \\
- \\
- \\
- \\
-\end{array}$ \\
\hline 24. & $\begin{array}{l}\text { I. } \mathrm{S} . \\
16 \text { meses } \\
51-623\end{array}$ & $\begin{array}{l}\mathrm{Dt} \\
\mathrm{T}\end{array}$ & $\begin{array}{l}1 \\
2\end{array}$ & $\begin{array}{l}6740 \\
\text { Falleció }\end{array}$ & $\delta^{o b .}$ & $\mathbf{3}$ & 117 & 5 & 20 & 54 & 84.5 & 0.63 & 35 & - & $\ldots$ \\
\hline 25. & $\begin{array}{l}\text { P. C. } \\
5 \text { meses } \\
50-5997\end{array}$ & $\begin{array}{l}\mathbf{A} \\
\mathrm{T}\end{array}$ & $\begin{array}{l}1 \\
4 \\
6\end{array}$ & $\begin{array}{l}2750 \\
3180 \\
3200\end{array}$ & $\begin{array}{c}\mathrm{Ob} . \\
\mathbf{N} \\
\mathbf{N}\end{array}$ & $\begin{array}{l}2 \\
1 \\
1\end{array}$ & $\begin{array}{l}120 \\
121 \\
126\end{array}$ & $\begin{array}{r}12 \\
3.3 \\
-\end{array}$ & $\begin{array}{l}17.4 \\
21.4 \\
21\end{array}$ & $\begin{array}{l}42 \\
41 \\
53\end{array}$ & $\begin{array}{l}81 \\
93 \\
90\end{array}$ & $\begin{array}{l}0.52 \\
0.74 \\
0.40\end{array}$ & $\begin{array}{c}49.2 \\
33.9 \\
-\end{array}$ & $\begin{array}{l}37 \\
-- \\
-\end{array}$ & $\begin{array}{l}12 \\
-\end{array}$ \\
\hline
\end{tabular}


De 23 niños en que se hizo determinación de cloro plasmático, en 12 se encontró éste descendido; en 6, normal y en 5, elevado. Respecto a cloro globular, que se determinó en 17 casos, en 8 estaba normal; en 7, aumentado y en 2, bajo. El índice clorémico se encontró normal en 2 de los 17 casos en que se investigó; en 14, elevado y en 1, descendido.

El potasio del suero se controló en 20 casos y halló normal sólo en 2 , aumentado en $10 \mathrm{y}$ bajo en 8 . Los valores extremos de este electrolito fueron $0.8 \mathrm{y}$ $12 \mathrm{mEq}$. Del Carril y Larguía lo encuentran bajo en 20 de 33 casos y alto en 13 y Darrow, en un total de 7 lactantes, normal en 2, aumentado en 3 y disminuído en 2. Este último autor refiere haber encontrado cifras altas, entre 10 a $12 \mathrm{mEq}$., en pacientes agónicos que no habían recibido tratamiento.

La proteinemia se encontró superior a $65 \mathrm{~g} \mathrm{o} / \mathrm{oo}$ en 17 de 23 casos y normal o disminuída en los restantes, resultados que en general coinciden con los comprobados por otros autores.

Si dirigimos, ahora, nuestra atención a los resultados del tratamiento, observaremos que en el grupo $\mathrm{A}$ fallecieron 3 de los 16 lactantes tratados y en el grupo $B, 2$ de los 9 que lo componían. Es también de interés hacer notar que en el primer grupo se obtuvo normalización de la hidratación desde el punto de vista clínico en un tiempo promedio de alrededor de 5 días $y$ en el segunda esto sólo liegó a conseguirse en 3 casos a los 8 días. Si nos detenemos en los resultados expuestos en Ias Tablas 2 y 3 resalta el hecho de no haberse conseguido normalización completa del ionograma al término del período de hidratación, tel que se extendió todo el tiempo estimado necesario para obtener dicho fin. Así, por ejemplo, en el grupo A, al llegar el tratamiento a su término, la natremia había ascendido en 9 enfermos y de éstos sólo en 3 alcanzado la normalidad, en 2 , uno de ellos con valor normal, ella había permanecido invariable y en 3 , descendido. 'En el grupo B, en 4 casos el $\mathrm{Na}$ ascendió con el tratamiento y en
3 descendió y no se completó la hidratación, no obstante el suministro de solución de Ringer en dosis suficiente. En los 2 casos restantes la concentración de $\mathrm{Na}$ sobrepasó los límites normales, $170 \mathrm{mEq}$. en uno de ellos, lo que, sin embargo, no tuvo tradueción clínica.

En el grupo A se usó en 12 casos la solución de Hartmann para combatir la acidosis y observó en todos efecto beneficioso, con normalización de la respiración en 7 de ellos, en que ésta era de tipo acidótico, en el plazo de 2 a 3 días. En 12 de estos niños existía compromiso sensorial variable de simple apatía a inconsciencia absoluta, el cual, en general, se mostraba más marcado mientras mayor era el déficit de bicarbonato. La recuperación de la consciencia se obtuvo en todos los casos, dentro de 2 a 3 días de iniciado el tratamiento, a lo que hizo excepción el caso 11 , que continuó inconsciente hasta el fallecimiento. En la totalidad de los niños la cifra de bicarbonato ascendió, pero sólo en 3 llegó a lo normal al tercer día, en 6 se alcanzó esto después de repetir la solución alcalinizante y en 4 ella no llegó a la normalidad.

Si se compara la evolución clínica de Ios casos con el volumen de solución de Hartmann empleado se comprueba que hubo rápida normalización de la consciencia cuando ésta se administró en dosis suficiente para restituir la totalidad del défícit calculado de bicarbonato. En los casos en que este resultado no se obtuvo, a pesar de procederse en igual forma, como sucedió en los casos 4 y 7 , ello se debio a la persistencia de los factores determinantes de la acidosis, tales como diarrea, fiebre, ayuno relativo, etc. En el grupo B el laboratorío reveló en 8 niños acidosìs, la que sólo en 2 pudo ser sospechada clínicamente. Usada en uno de éstos solución de Hartmann en dosis suficiente, se obturo rápida recuperación. De esta serie fallecieron 2 lactantes, ambos tratados con solución de Ringer, suero glucosado ai $10 \%$ y plasma. En ambos el clínico no planteó la posibilidad de acidosis y no 
usó, por consiguiente, solución de Hartmann. Sin pretender que los niños hubieran podido salvarse, creemos que el empleo de la solución antedicha, dada la respuesta satisfactoria de casos semejantes, habría sido de claro beneficio.

Un caso que merece comentario especial es el 23, el cual, con reserva alcalina inicial de $23 \mathrm{mEq}$., su equilibrio ácido-básico se desplazó en el sentido de la franca aicalosis hasta alcanzar a 39 mEq. $\left(85.8\right.$ vol. de $\left.\mathrm{CO}^{2} \%\right)$ durante la administración de solución de Ringer con vuelta de él a la normalidad con la suspensión de ésta. La explicación de este fenómeno la da Darrow, quien ha observado casos semejantes. Este autor sostiene que el tratamiento de un cuadro de diarrea profusa con sales de $\mathrm{Na}$ puede llegar a la alcalosis por intervención de un mecanismo renal, el que se desencadenaría ante abundancia de iones $\mathrm{Na} \mathrm{y} \mathrm{Cl}$ y deficiencia de $\mathrm{K}$, ya que la concentración del $\mathrm{HCO}_{3}$ del suero varía en proporción directa a la del Na intracelular e inversa a la del $K$ de los músculos.

A continuación, resumimos 3 observaciones, que ilustran sobre las alternativas del tratamiento.

CASO 5. D. C. Obs. $51 / 2288$. Lactante de s meses. Peso: 4,560 g.

Desde hace 2 semanas presenta deposiciones líquidas, mucosas, 8 a 10 al día, $y$, ocasionalmente, vónitos, cuadro que se ha acentuado en los últimos 3 meses, en que se agrega decaimlento, tendencia al sueño y sed intensa. Nactó de parto gemelar, con peso de 2,400 g. A los 4 meses raquitismo. Al ingreso se comprueba un ntño enflaquecido, febril, semi-inconsciente y en posición de esgrimista. Piel muy deshidratada, en algunas zonas empastada, paniculo escaso, mucosas secas y ojos hundidos. Tórax largo, blando, con rosario costal discreto. Respiraciones profundas y frecuentes. Extremidades frías y clanóticas.

A la llegada del nlño al Servicio se comprueba reserva alcalina de $8 \mathrm{mg} q$. (17.6 $\mathrm{rol}$. de $\mathrm{CO} 2 \%)$, ligero aumento del índice clorémico, hiponatremia y leve aumento del potasio. Habia, además, hemoconcentración revelada por marcada niperprotelnemia y elevación del volumen globular. Los liquidos totales se calcularon en 3,150 ce y a base de un déficit de $\mathrm{CO} 2$ de $19 \mathrm{mEq}$. se supuso el déficit total de este catión en alrededor de
$60 \mathrm{mEq}$. Se restituyó sólo la mitad de esta en forma de 180 be de solu:zón de Hartmann mezclada con igual volumen de suero glucosado al $10 \%$ por vía, endovenasa, gota a gota, Posteriormente, se continuó con soluclón de Ringer en dosis suficiente para reparar el cléficit de $\mathrm{Na}$ calculado en $40 \mathrm{mEa}$. Al día siguiente el niño amanece consclente, si bien a ratos se le nota soporoso, las respiraciones han dismlnuído en frecuencia y se aprecia al examen mejor hldrataclón, qua se confirma por un aumento de peso de $340 \mathrm{~g}$. Este día se continúa la administración de . suero Ringer por fleboclisis y per as e Inicla la realimentación. Al tercer día el sensorio está totalmente despejado. Entre atros exámëntes el de deposición revela mucus, pus y sangre y Kebsiella al cultivo. A esta altura de la evolución el laboratorio da cuenta de aumento apreciable de las cifras de $\mathrm{Na}$ y reserva alcalina y descenso de la proteinemia. Se sigue con soluciones de Hartmann y Ringer por via oral, que se suministran durante otros 3 días. En el control sanguíneo del séptimo día el Na está normal, la reserva alcalina algo descendida $\mathrm{y}$ el $\mathrm{Cl}$ y $\mathbf{K}$ persisten ligeramente alterados. La recuperación clínica del lactante avanza y a los 15 días alcanza un peso de 5,720 g. Este efemplo muestra en forma clara la posibilfdad práctica de condicionar las indicaciones terapéuticas a las determinaclones de laboratorio.

\section{CASO 9. A. B. Obs. $51 / 1245$. Lactante de} 2 meses. Peso: $3,140 \mathrm{gg}$.

Desde hace 9 dias presenta deposiciones frecuentes, mucosas y, además, en los últímos días, vámitos. Niño decaído, pálido, en deficiente estado nutritivo, deshiäratado, con persistencla de pliegues en abdomen. Mucosas bucales secas. Hipotonía muscular. El $\mathrm{Na}, \mathrm{Cl}$ y $\mathrm{K}$ se comprueban aumentados y la reserva alcalina disminuida. Este cuadro de hiperelectrolitemia colnclde con un estado de hemoconcentración, ello revelado por cifras de proteinas y hematocrito elevadas. Se administra solución de Hartmann por vía endovenosa para reemplazar el déficlt calculam do de $\operatorname{CO} 2 \mathrm{~g}$, además, no obstante los valores altos de $\mathrm{Na}$ y $\mathrm{Cl}$, de suero Ringer, con el fin de corregir la intensa deshidratación. Posterlormente, la hidrataclón se sigue con el suministro de soluciones de Hartmann $y$ Ringer. El caso evoluclona clínicamente en forma satisfactoria, los electrolitos se aproximan a la normalidad $y$ el peso experimenta notable aumento. Los pormenores del tratamiento pueden apreciarse en la Tabla 2.

Rapoport20 ha observado casos como éste con hiperelectrolitemia inicial, situación a la que también puede llegarse experimentalmente por la ingestion insuficiente de agua 0 inyección de soluciones salinas hipertóntcas 
o de urea, como lo han demostrado Elkinton y colaboradores en perros:1. Es indudable que en nuestro caso, por causas que desconosemos, la pérdida de agua no se acompañó de la pérdida proporcional de sales. El suministro de líquidos hidrató el compartimento extracelular, io que trajo por consecuencia la desaparición de la hemoconcentración (descenso de la proteinemla y normaltzación de los valores de $\mathrm{Na}$ y $\mathrm{CI}$. El hecho de que no apareciere edema, a pesar del exceso de sales administradas, cabe seguramente atribuirlo al buen funcionamlento de los mecanismos reguladores, en especial, renales.

CABO 19. A. S. Obs. $51 / 117$, Niño de 2 meses. Peso: $3,400 \mathrm{~g}$.

Desde 10 días antes deposiclones liquldas, frecuentes, algunas sanguinolentas No ha presentado vómitos. Al ingreso se halla un lactante en mal estado nutritivo, decaído. Piel pálida, con elasticidad y turgor muy dlsminuídos. Mucosa bucal seca. Se indica dieta hidrica de 12 horas, transfuslón sanguinea de 60 cc y fleboclisis de suero Ringer y glucosado al $10 \%$, en partes iguales, $240 \mathrm{cc}$ de cada uno, pero por tropiezos técnicos sólo recibe la mitad de estas solucfones. A contznuación se sigue con meacla hidratante $y$, Iuego, inicla realimentación con leche humana descremada. Durante el segundo tercer días se repite la fleboclisls $\mathrm{y}$ agrega plasmoterapia de 80 cc. El examen de deposiciones demuestra mucus y pus y bacílos piociánilcos al cuitivo. En los dias siguientes se completa la ración liqulda con $40 \mathrm{cc}$ de mezcla de suero Ringer y agua de canela con $6 \%$ de maltosa-dextrina. Durante este tlempo el niño sigue decaído, con deposlciones frecuentes, alteradas y presenta fiebre. En vista que su hidratación no mejorara al séptimo y octavo días, se suministra de nuevo solución Ringer, 250 y $200 \mathrm{cc}$, respectivamente, con i.gual volumen de suero glucosado al $10 \%$, a pesar de lo cual no se aprecla recuperación subjetiva, la deshidratación persiste y aparece hipertonía de las extremidades superiores $\mathrm{y}$ músculos dorsales y pectorales. Liquido céfalo raquídeo transparente, con $0.60 \mathrm{~g} \%$ de albrimina, Pandy y Nonne Appelt $t, 2$ linfocitos por mmi y glucosa y cloruras nonmales. En adelante, el mal estado general del niño se acentúa, reclbe la allmentacion en forma deficiente, aparece bronconeumonía y fallece, esto a Jos 15 días तe su ingreso.

En este caso, perteneciente al grupo B, paralelamente al tratamiento y sin que el médico tratante lo supiera, hicimos determinación de electrolitos. Al ingreso había natremia normal. aumento del ínalice clorémico, reserva alcalina muy descendida. ligero aumento de la kalemla y proteínas del suero. En exámenes de control al tercer dia se apreció descenso del $\mathrm{Na}$, reserva alcalína $\mathrm{y} \mathbf{K}$ y al octavo día, en relación con el cuadro de hipertonia, el Na se encontró cercano a lo normal, el Cl a indice clorémico disminuídos, la kalemia ligeramente descendida y la reserva alcalina siempre mug baja. En este caso está fuera de dudas el gran beneficio que habría derivado del empleo de la solulución de Hartmann. Como se vió, la intensa acldosis presente no se corrigio con las soluciones usadas. Un hecho que llama la atención es la existencia, al ingreso, de in. tensa deshidratación sin ir acompañada de gran hemoconcentración ni hiponatremia. Resulta claro, de los exámenes practicados, que con el tratamiento impuesto no se consiguió hidratación ni restitución adecuadas del déficit de electrolitos.

En cuanto a las variaciones del $\mathrm{K}$ del suero, el nivel de este catión experimentó marcadas fluctuaciones, especialmente tendencia al aumento, hasta alcanzar a veces cifras elevadas (10 y $12 \mathrm{mEq}$.), como en los casos 1 y 8 , sin llegar a observarse, felizmente, manifestaciones tóxicas. En la literatura se describen casos de intoxicación, entre ellos el relatado por Govan ${ }^{2 *}$, debido a la administración subcutánea de solución de Darrow y que se manifestó por shock, encontrándose en ese momento una kalemia de $12.3 \mathrm{mEq}$. Finch, Sawyer, y Flinn"*, por su parte, han encontrado que un nivel sanguíneo de 10 a $15 \mathrm{mEq}$. de $\mathrm{K}$ es letal. Darrow expresa que una concentración de $\mathrm{K}$ en el suero de 10 a $12 \mathrm{mEq}$. puede llegar a producir bloqueo cardiaco, compromiso relacionado con el aumento extracelular de este catión $y$ no con su nivel intracelular en el corazón.

Como más arriba se dijo, en 8 casos se halló hipokalemia, uno de éstos con cifra de $0.8 \mathrm{mEq}$. Ninguno de los niños, sin embargo, mostró la sintomatologia que suele acompañar a tal signo, a saber, parálisis musculares, dilatación cardiaca y alteraciones del electrocardiograma. Cifras tan bajas, como las observadas por nosotros, han sido encontradas por varios autores, entre ellos Gamble, quien halló una concentración de $1.3 \mathrm{mEq}$, en un lactante con diarrea intensa. Robinson"* ha dado cuenta igualmentry de valores de 0.58 a 1.3 mEq. en 4 casos de gastroenteritis aguda, todos los cuales fallecieron. 
Nosotros usamos solución de Darrow sólo en 3 casos, con valores de $0.8,2.3$ y $2.5 \mathrm{mEq}$. de $\mathrm{K}$ y sólo en uno de ellos comprobamos corrección del déficit. A este propósito quizás sea del caso citar a Nassau ${ }^{25}$, autor que declara no haber observado efectos brillantes con el empleo de la solución antes mencionada. Karelitz ${ }^{26}$, por su parte, también con experiencia en este sentido, no se pronuncia en dicho respecto y se limita a expresar que en sus manos ella no ha demostrado efectos nocivos.

\section{Resumen.}

En vista de la alta importancia hoy en día adquirida por el déficit de electrolitos en los trastornos nutritivos agudos y su adecuada restitución con soluciones salinas, se elabora y realiza un plan de trabajo que contempla la determinación de ellos en lactantes aquejados de dispepsia o toxicosis. El estudio de laboratorio comprendió la determinación de sodio, cloro, potasio y reserva alcalina $\mathrm{y}$, además, proteinemia, cuyos niveles se usaron como guía en la dirección de la terapéutica de restitución hidrosalina a base de las soluciones de Ringer, Hartmann y Darrow. El trabajo abarcó en total 25 casos, 16 en un grupo en que el tratamiento se condicionó a los resultados de laboratorio $\mathrm{y}$ 9 en otro que sirvió de control y en el que las determinaciones permitieron juzgar del mayor o menor acierto del clinico en sus medidas terapéuticas.

Las determinaciones iniciales de sodio hechas en 24 casos revelaron hiponatremia en 19, hipernatremia en 3 y valores normales en 2 . No se comprobó un paralelismo estrecho entre grado de hiponatremia y deshidratación. En 25 casos se determinó la reserva alcalina y encontró baja, con cifras inferiores a $23 \mathrm{mEq}$, en 22 y normal en 3 . En 15 niños con toxicosís había una disminución marcada del bicarbonato. Es interesante hacer notar que de los 21 lactantes en que el laboratorio dió al diagnóstico de acidosis, sólo en 9 de ellos pudo el clínico sospechar este estado por la existencia de respiración acidótica, sin que los ca- sos restantes presentaran este signo ni con cifras de reserva alcalina extremadamente bajas. El potasio del suero se determinó en 20 niños y halló aumentado en 10 , bajo en 8 y normal en 2 .

En el primer grupo de niños, en el que las medidas de hidratación estuvieron sujetas a los resultados de las determinaciones de laboratorio, 3 fallecieron y en los restantes se obtuvo recuperación completa del estado de deshidratación en un tiempo promedio de alrededor de 5 días, a diferencia de los 9 lactantes del grupo de control, de los cuales, excluidos 2 que sucumbieron, sólo en 3 se consiguió igual resultado en un plazo de 8 días. En el primer grupo se usó en 13 solución de Hartmann para corregir el déficit de bicarbonato y observó en todos, excepto 1, efecto beneficioso rápido, tanto en la normalización de la respiración, cuando ésta era de tipo acidótico, como en la recuperación de la consciencia. En el segundo grupo, en el que 8 de los 9 lactantes presentaban acidosis, sólo en 1 de 2 en que ésta pudo ser sospechada clínicamente, se empleó dicha solución igualmente con buenos resultados.

En cuanto a las fluctuaciones del potasio del suero, en apreciable número de casos se comprobó tendencia a aumento de este catión en el curso de la terapéutica de hidratación, con valores, en algunos niños, entre 10 a $12 \mathrm{mEq}$., sin comprobarse, sin embargo, manifestaciones clínicas tóxicas. En 3 casos, en que por hipokalemia acentuada usó solución de Darrow, sólo en 1 observó corrección del déficit.

\section{Summaiy.}

A study on electrolyte concentration in acute nutritional disturbances is carried out. The work comprised sodium, chloride, potassium and bicarbonate determinations on 25 infants suffering from dyspepsia or toxicosis, 16 of them in a group where the electrolyte figures provided a criteria for replacement treatment and 9 in another group, taken as control. As 
replacement therapy Ringer, Hartmann, Darrow and glucose solutions were used.

Sodium analysis on admission on 24 infants revealed decreased levels in 19 , high in 3 and normal in 2. A close relation was not seen between deficiency of this electrolyte and dehydration. Of 25 patients 22 had acidosis, with bicarbonate figures lower than $23 \mathrm{mEq}$, and 3 normal acid-base balance. Infants affected with toxicosis showed the highest electrolyte deficits. In the first group, except 3 patients who died, all of them had a complete recovery of dehydration in a period of about 5 days and in the second group, excluding 2 fatalities, 8 days must elapse before seeing the same result in 3 patients. Attempts to restore potassium losses by Darrow's solution did not have striking results.

\section{Bibliografia.}

1. WIEISERHOLD, A. - Fl equilibrio àcidobásico en Pediatria. Tesis de Prueba. Imprenta Continental. Santiago de Chille. 1927.

2.-COFRE, P. - Contribución al estudio humoral $y$ a la terapéutica de la toxleosis. Tesis de Prueba. Bantiago de Chile. 1938.

3.-AMENABAR, E. - Contribución al estudio humoral en las deshidrataciones agudas del Iactante. Tesis de Prueba. Talleres Gráficos El Chileno. Santiago. 1943.

4.-ARIZTIA, A. - Consideraciones sobre el tratamiento de la deshidratación aguda o exícosis del Iactante. A.rch. Arg. de Pediatria 19: 83, 1943.

5.-GAMBLE, J. - Deficits in diarrhea. J. Pediat, 30: 448, 1947.

6.-GAMBLE, J. - Chemieal Anatomy, Physíology and Pathology of extracelular fluids. Harvard Medical school. 1941.

7.-DARROW, D. - Body fluid physiology; relation of tissue compasition to problems of water and electrolyte balance. New England J. Med. 233: 91, 1945.

8.-DARROW, D. y colaboradores. - Disturbances of water and electrolytes in infantile diarrhea. Pediatries 3: 129, 1949.

D.-DARRIOW, D. - Disturbance in electrolyte metabolisin in man and their ma- nagement. Bull. New York Acad. Med. 24: $147,1948$.

10.-DARROW, D. - Retention of electrolyte during recovery from severe dehydration due to diarrhea. J. Pediat. 28: 515, 1946.

11,-HARTMANN, A. y colaboradores. Treatment of diabetic acidasis. J. Pediat. 31: 274, 1947.

12.-BUTLER, A. y TALBOT, N. - Parenteral fluid therapy. New England. J. Med. 231: 585,1944 .

13.-FANCONI, G. - Zur Pathogenese und Theraple der Toxikosen, mit besonderer. Berïcksichtigung der Sauglingstoxicose. Helvet. Paediat. Acta 4: 475, 1949.

14.-FANCONI, G. y ROSSI, F. - Zur Pathogenese und Therapie der Toxicosen. Helvet. Paediat. Acta 2: 305, 1947.

15. CORONA, L. - Química normal y patológica de la sangre, Ed, Ercllla. Santiago de Chile. 1942.

16.-HAND, A. y LEININGIER, C. - Parenteral fluid therapy in children Medical Clinics of North America 34: 53, 1950.

17.-GOVAN, C. y DARROW, D. - Use of potassium chloride in the treatment of the dehydration of diarrhea in infants. J. Pediat. 28: 541, 1946.

18. $D$ DHEN, P. y DUBOW, E. - Recent advances in parenteral therapy in Pediatrles. American Practitioner 1: 3, 1950.

19.-DEL CARRT, M. y LARGUIA, A. - Desbldratation en Pediatría. $\mathrm{R}$ Ateneo. Buenos Aires. 1945.

20.-RAPOPORT, is. - Hyperosmolarity and hyperelectrolytemia in pathologic conditions of childhood. Am. J. Dis. Childa. 74: 682, 1947.

21.-ELKINTON, J.; DANOWSKI, T. y WINKLER, A. - Hemodynamic changes in salt depletion and in dehydration. J. Clin. Investigation 25: 120, 1946.

22. GOVAN, C. y WEISETH, W. - Potasslum intoxication. J, Pediat. 28: 550 , 1946.

23.-FINCH, C.; SAWYHR, C. Y FLINN, J. clinical syndrome of potassium intoxication. Am. J. Med. 1: 337, 1946.

24.-ROBINSON, P. - Potassium in acute gastroenteritis. Ann. Paediat. 153: 157, 1939.

25.-NASISAU, E - tuber toxische Gastro-Enteritiden. Ann. Paediat. 176: $82,1851$.

26. -KARELTTZ, S. - Alimentary toxicosis. Rev. Chilena de Ped. 18; 911, 1047.

(Los autores agradeczn ai Dr Carlos Garcés. Jefe del Laboratorio, sa paliosa ayuda en la realización de este estudio). 